GJI Geomagnetism, Rock Magnetism and Palaeomagnetism

\title{
Tangential stress at the core-mantle interface
}

\author{
D. Jault \\ Univ. Grenoble Alpes, Univ. Savoie Mont-Blanc, CNRS, IFSTTAR, IRD, ISTerre, 38000 Grenoble, France. E-mail: dominique.jault@univ-grenoble-alpes.fr
}

Accepted 2020 January 25. Received 2019 December 3; in original form 2019 July 17

\begin{abstract}
SUMMAR Y
Fluctuations in the rotation rate of the solid Earth over periods from 5 to $100 \mathrm{yr}$ result from exchanges of angular momentum between the fluid outer core and the solid mantle. The coupling mechanism mediating angular momentum transfer is not clear yet. Here, I revisit local Cartesian models for the pressure stress on a bumpy core-mantle interface. One common approach consists in analysing forced magnetohydrodynamic modes arising from the interaction between a steady flow along the core-mantle interface and boundary topography. The wave amplitude scales as the height $\zeta$ of corrugations and the pressure stress as $\zeta^{2}$. As expected from Newton's third law, the tangential stress on the fluid is opposite to the tangential stress on the solid. It is exactly compensated by non-zero mean electromagnetic and Coriolis forces, which both result from interactions at infinity and not with the electrically insulating solid. Requiring zero net flux of mass and electrical current at infinity in order to better model closed systems necessitates to restore mean flow acceleration. This makes possible to investigate whether there is momentum transfer into the fluid interior or instead dissipation next to the boundary. Fluid stratification enhances the horizontal stress exerted by the pressure field on the core-mantle boundary but we have yet to describe the mechanism to transport momentum from the boundary into the fluid.
\end{abstract}

Key words: Core; Earth rotation variations.

\section{INTRODUCTION}

It has long been known that exchanges of angular momentum between the Earth's fluid core and solid mantle give rise to the fluctuations in the Length Of the Day (LOD), a few milliseconds in amplitude, that are observed over the period range 5-100 yr (Hide 1977; Jault et al. 1988; Bärenzung et al. 2018). However, the torques acting between core and mantle that bring about these angular momentum exchanges are still debated (Roberts \& Aurnou 2012; Glane \& Buffett 2018).

The strength of the topographic torque produced by dynamical pressure forces acting on irregularities of the core-mantle interface is particularly uncertain. There were attempts at estimating this torque directly from models of the topography and fluid pressure at the core-mantle boundary (Hide 1989; Jault \& Le Mouël 1990). Fluid pressure with typical amplitude of about $10^{3} \mathrm{~N} \mathrm{~m}^{-2}$ was obtained from core surface velocity models assuming tangential geostrophy at the core surface. These models have now been superseded by quasi-geostrophic models (Pais \& Jault 2008; Amit \& Pais 2013) and the topography at the core-mantle interface remains poorly known (Schlaphorst et al. 2016). Kuang \& Chao (2001) estimated the topographic torque at the core-mantle boundary from its value in a homogeneous dynamo model and found it too weak to account for the observed LOD variations.

Core-mantle coupling may depend on properties of the core next to the mantle that are not well reproduced in numerical models of the geodynamo, although our information about these properties for the Earth's core is also inadequate, see the debate about stratification of the upper layers of the core (Helffrich \& Kaneshima 2010; Irving et al. 2018). Clearly, theoretical studies may help us to find out what are the key core and mantle properties that determine the strength of the coupling. Various local studies of pressure torque were carried out. Assuming that the fluid at the top of the core flows with a uniform velocity $U_{0}$ relative to the solid, the average stress is calculated in the form $\langle F\rangle=$ $K U_{0}$ (Hide 1977; Lambeck 1980). The coefficient $K$ can then be fed to global models. The aim of such studies is to determine how $K$ varies with model parameters.

We may learn from studies of the 'gravity wave torque' that is responsible for part of the angular momentum transfer between winds in the atmosphere and the solid Earth. These investigations aim at parametrizing orographic wave drags. Actually, the gravity wave torque is defined as the part of the 'mountain torque' (i.e. the pressure torque) on the solid Earth that is unresolved in global atmospheric reanalysis projects (Egger et al. 2007). For future reference, it is noteworthy that most of the global mountain torque is cancelled out (at periods over $40 \mathrm{~d}$ ) by the friction torque. The importance of the gravity waves torque results from the ability of these waves to transfer vertically a mean 
stress over large heights, in the absence of dissipation (McIntyre 1980). The mean stress scales linearly with the basic state flow $U_{0}$ and the buoyancy frequency $N$ and quadratically with the topography height (Palmer et al. 1986).

Similarly to these atmospheric studies, Braginsky (1998) investigated topographic core-mantle coupling in the plane tangent to the boundary. He calculated the tangential pressure stress on a bumpy interface between a stratified fluid layer and an insulating solid in the presence of a transverse magnetic field. The tangential stress stems from evanescent modes attached to the boundary rather than from gravity waves propagating deep into the core. The corrugations on the interface, in this model, have wavelength a significant fraction of the core radius, typically $L \sim 800 \mathrm{~km}$. Therefore, he had to account for the spherical geometry of the core-mantle boundary and incorporated a $\beta$-effect in a quasi-geostrophic model. He found that tangential stress linearly increases with buoyancy frequency $N$ and that it scales with velocity $U_{0}$ as $\sqrt{U_{0}}$ and with topography height $\zeta$ as $\zeta^{2}$. Glane \& Buffett (2018) investigated small-scale rugosity of the core-mantle boundary. They also found that the tangential stress is dramatically enhanced if the uppermost core is strongly stratified and the boundary is spanned by a transverse magnetic field. They concluded that a topography with height $\simeq 50 \mathrm{~m}$ and wavelength $L \sim 100 \mathrm{~km}$ yields a tangential stress that is sufficient to account for the length of the day observations if the buoyancy frequency in the stratified layer is of the order of the rotation frequency or larger. Such a topography is so small that we cannot hope to detect it with seismic waves.

I revisit several Cartesian models of pressure stress on a bumpy horizontal interface between a conducting fluid and an electrically insulating solid. The entire set-up is rotating about a vertical axis and permeated by a uniform magnetic field. In all these models, the velocity and magnetic field perturbations on the fluid side are confined close to the fluid-solid interface. This feature justifies the investigation of a local Cartesian model. The confinement results either from the orientation of the magnetic field parallel to the fluid-solid interface (Anufriev \& Braginskii 1975; Moffatt 1977; Hassan \& Eltayeb 1982) or from the fluid stratification (Braginsky 1998; Glane \& Buffett 2018). These studies consist in the calculation of linear modes forced by the boundary topography, of height $\zeta$. Hence, their amplitude is $O(\zeta)$ also, and interaction between the pressure field of these modes and the topography results in a spatially averaged tangential stress on the boundary, which scales as $\zeta^{2}$.

Although the solid boundary is electrically insulating, the mean electromagnetic force parallel to $U_{0}$ acting on the fluid half space needs not be zero if a net electric current flux leaves the system. Similarly, the Coriolis force contributes to the net stress acting on the fluid along $U_{0}$ if there is a net volume flux transverse to $U_{0}$ leaving the system. Actually, the previously cited Cartesian models of tangential stress did not preclude a net flow in the direction orthogonal to the mainstream flow $U_{0}$ and the boundary undulations. Hence, a mean Coriolis force arises that cannot be neglected in the balance of forces acting on the fluid. This contrasts with plane layer dynamo models, where the vertically integrated mass transport is assumed to be zero (Abdel-Aziz \& Jones 1988). Similarly to dynamo models and by analogy with the sphere, I will seek to impose no net mass flux and electrical current at infinity. This necessitates to introduce $O\left(\zeta^{2}\right)$ time derivatives of the spatially averaged flow and magnetic field.

In all the models discussed here, the core-mantle boundary is locally approximated by a plane with sinusoidal undulations. The fluid is inviscid and the solid is non-conducting, cancelling both the viscous and electromagnetic stresses on the solid wall. In addition, the governing equations are linearized. I present the common features of these models in Section 2, where I also give expressions for each term of tangential stress on the boundary. In the remaining of the text, I use these expressions for two different models and show that the tangential stress terms on the fluid add up to zero. I recapitulate the model developed by both Anufriev \& Braginskii (1975) and Moffatt (1977) in Section 3. The model of Braginsky (1998) and Glane \& Buffett (2018), which incorporates stable stratification, is discussed in Section 4. Although the last two studies apply for two distinct asymptotic limits of the model, they broadly agree for typical geophysical values. I discuss the transfer of momentum from the boundary into the fluid in Section 5, where I describe the vertical propagation of inertial waves and the concomitant momentum transport for the special case of horizontal imposed magnetic field. The paper ends with concluding remarks.

\section{TANGENTIAL STRESSES ON EITHER SIDE OF THE FLUID-SOLID INTERFACE}

\subsection{Linearized equations}

An approximately plane interface $z=\zeta(x)$ separates an electrically conducting fluid $(z<\zeta)$ from a non-conducting solid ( $z \geq \zeta)$. The entire set-up rotates about the $z$-axis with angular velocity $\Omega$. Far from the boundary, the fluid moves with velocity $U_{0}$ in the $x$ direction. A uniform magnetic field pervades both the fluid and the solid regions. The topography is considered as 1-D:

$\zeta(x)=\Re\left(\hat{\zeta} \exp \left(i k_{x} x\right)\right)$

where $k_{x}$ is a positive real number, $\left|k_{x} \zeta\right| \ll 1$, and $\Re$ means taking the real part of the complex expression. The unit vectors in the $x, y$ and $z$ directions are $\mathbf{e}_{x}, \mathbf{e}_{y}$ and $\mathbf{e}_{z}$ respectively.

The models I discuss here were designed to have stationary velocity and magnetic field solutions, $\mathbf{u}$ and $\mathbf{b}$. As a result, the terms $\partial \mathbf{u} / \partial t$ and $\partial \mathbf{b} / \partial t$ are neglected in the momentum and induction equations respectively. The imposed magnetic field $B_{0} \mathbf{e}_{B}$ is uniform in directions that differ between models. The governing equations in the fluid region, after linearization, are:

$$
\begin{aligned}
& \frac{\partial \mathbf{u}}{\partial x}+2 R o^{-1} \mathbf{e}_{z} \times \mathbf{u}=-\nabla p+A l^{-1} \mathbf{e}_{B} \cdot \nabla \mathbf{b}, \\
& A l^{-1} \mathbf{e}_{B} \cdot \nabla \mathbf{u}-\frac{\partial \mathbf{b}}{\partial x}+R_{m}^{-1} \nabla^{2} \mathbf{b}=0
\end{aligned}
$$


$\nabla \cdot \mathbf{b}=0, \quad \nabla \cdot \mathbf{u}=0$

where $p$ is the total pressure. The Lorentz force $\mathbf{j} \times \mathbf{B}$ (with $\mathbf{j}$ the electrical current density, and $\mathbf{B}=B_{0} \mathbf{e}_{B}+\mathbf{b}$ ) breaks down into the Maxwell stress term $\mathbf{B} \cdot \nabla \mathbf{B} / \mu$ and the magnetic contribution $-\nabla\left(B^{2} / 2 \mu\right)$ to the pressure force $(\mu$ is the magnetic permittivity). The length, velocity and magnetic field units are the horizontal scale of the bumps $k_{x}^{-1}$, the imposed velocity $U_{0}$ and $\sqrt{\rho_{0} \mu} U_{0}$ respectively ( $\rho_{0}$ is the fluid density). The pressure unit is $\rho_{0} U_{0}^{2}$. The Rossby number $R o$, the magnetic Reynolds number $R_{m}$ and the Alfvén number $A l$ are defined as $k_{x} U_{0} / \Omega, U_{0} / \eta k_{x}$ and $\sqrt{\rho_{0} \mu} U_{0} / B_{0}$, respectively ( $\eta$ is the fluid magnetic diffusivity). The number $Q=A l^{2} / R_{0} R_{m}$ introduced in this context by Moffatt (1977) will play a major role in the discussion. It corresponds to the inverse of the Elsasser number. With this choice of units, eq. (1) transforms into

$\zeta(x)=\Re(\hat{\zeta} \exp (\mathrm{i} x))$

The outward unit normal to the fluid region is given by

$\mathbf{n}=(-\mathrm{d} \zeta / \mathrm{d} x, 0,1) / \sqrt{1+(\mathrm{d} \zeta / \mathrm{d} x)^{2}}=(-\mathrm{d} \zeta / \mathrm{d} x, 0,1)$

because we assume the topography to be small in comparison with the wavelength. In the presence of a steady flow relative to the boundary $\mathbf{e}_{x}$ the linearized expression for the no penetration condition becomes

$\left.u_{z}\right|_{z=0}=\frac{\mathrm{d} \zeta}{\mathrm{d} x}$.

Velocity and magnetic field solutions are sought in the form

$\mathbf{u}(x, z)=\Re(\hat{\mathbf{u}}(z) \exp (\mathrm{i} x)), \quad \mathbf{b}(x, z)=\Re(\hat{\mathbf{b}}(z) \exp (\mathrm{i} x))$,

since they have the same dependence on $x$ as the boundary topography. Eq. (7) transforms into

$\left.\hat{u}_{z}\right|_{z=0}=\mathrm{i} \hat{\zeta}$

The magnetic field is a harmonic potential field in the solid region $z \geq \zeta(x)$ :

$\mathbf{b}_{m}=-\nabla \psi, \quad \psi=\Re(\hat{\psi} \exp (\mathrm{i} x-z))$.

It is continuous at the fluid-solid interface and subject to the condition

$\hat{b}_{x}=-\mathrm{i} \hat{\psi}, \quad \hat{b}_{y}=0 \quad \hat{b}_{z}=\hat{\psi}$

at $(z=0)$. Finally, because we have kept only linear terms in the equations, we can make the ansatz

$\mathbf{u}(x, z)=\Re\left(\hat{\mathbf{u}} \exp \left(\mathrm{i}\left(x+k_{z} z\right)\right), \quad \mathbf{b}(x, z)=\Re\left(\hat{\mathbf{b}} \exp \left(\mathrm{i}\left(x+k_{z} z\right)\right)\right.\right.$,

in the fluid region. Here, the $z$-wavenumber $k_{z}$ may take complex values.

\subsection{Tangential pressure stress on the solid}

We calculate the average pressure stress on the solid for all investigated models. It can be obtained as

$$
\begin{aligned}
\left\langle F_{p}\right\rangle & =\left\langle p \mathbf{n} \cdot \mathbf{e}_{x}\right\rangle=-\left\langle p \frac{\mathrm{d} \zeta}{\mathrm{d} x}\right\rangle \\
& =-\left\langle\left(\frac{\hat{p} \exp (\mathrm{i} x)+\hat{p}^{*} \exp (-\mathrm{i} x)}{2}\right)\left(\frac{\mathrm{i} \hat{\zeta} \exp (\mathrm{i} x)-\mathrm{i} \hat{\zeta}^{*} \exp (-\mathrm{i} x)}{2}\right)\right\rangle \\
& =\frac{\mathrm{i}}{4}\left(\hat{p} \hat{\zeta}^{*}-\hat{p}^{*} \hat{\zeta}\right)
\end{aligned}
$$

where the starred superscript denotes the complex conjugate. The expression can be further simplified by assuming that $\hat{\zeta}$ is real $\left(\hat{\zeta}=\hat{\zeta}^{*}\right)$.

The Lorentz force $\mathbf{j} \times \mathbf{B}$ breaks down into the Maxwell stress term $\mathbf{B} \cdot \nabla \mathbf{B} / \mu_{0}$ and the magnetic contribution $-\nabla\left(B^{2} / 2 \mu_{0}\right)$ to the pressure force. For an insulating boundary, the Maxwell stress $\mathbf{B} \cdot \nabla \mathbf{B} / \mu$ and the magnetic pressure stress on the solid region cancel each other out. It is thus necessary either to subtract the magnetic pressure from the total pressure before calculating the pressure stress or to add the contribution of the $\mathbf{B} \cdot \nabla \mathbf{B} / \mu$ term to the total pressure contribution. The average $x$-component of the Maxwell stress on the solid region is

$\left\langle F_{M}\right\rangle=-\left\langle\mathbf{e}_{x} \cdot\left(\left(\left(\frac{\mathbf{e}_{B}}{A l}+\mathbf{b}\right) \otimes\left(\frac{\mathbf{e}_{B}}{A l}+\mathbf{b}\right)\right) \cdot \mathbf{n}\right)\right\rangle$. 
When $\mathbf{B}_{0}$ is horizontal $\left(\mathbf{e}_{B}=\mathbf{e}_{x}\right)$, we have

$$
\begin{aligned}
\left\langle F_{M}\right\rangle & =\frac{1}{A l}\left(2\left\langle b_{x} \frac{\mathrm{d} \zeta}{\mathrm{d} x}\right\rangle-\left\langle\zeta \frac{\partial b_{z}}{\partial z}\right\rangle\right)=\frac{1}{A l}\left(2\left\langle b_{x} \frac{\mathrm{d} \zeta}{\mathrm{d} x}\right\rangle+\left\langle\zeta \frac{\partial b_{x}}{\partial x}\right\rangle\right) \\
& =\frac{\mathrm{i}\left(\hat{\zeta} \hat{b}_{x}^{*}-\hat{\zeta}^{*} \hat{b}_{x}\right)}{4 A l}=-\frac{\left(\hat{\psi} \hat{\zeta}^{*}+\hat{\psi} * \hat{\zeta}\right)}{4 A l},
\end{aligned}
$$

using (4) and the first identity (11). There is a factor two difference with Moffatt (1977), where the term proportional to $\partial b_{z} / \partial z$ above was omitted. We derive this term from a linearization of the vertical magnetic field,

$\left.b_{z}\right|_{z=\zeta}=\left.b_{z}\right|_{z=0}+\left.\zeta \frac{\partial b_{z}}{\partial z}\right|_{z=0^{+}}$.

This requires $k_{z} \zeta \ll 1$. The Lorentz force contributes also to the pressure term and the magnetic pressure stress has to be added to $\left\langle F_{M}\right\rangle$ to obtain the net action of the electromagnetic forces on the solid region. We have

$p_{M}=\frac{1}{2}\left|\frac{\mathbf{e}_{x}}{A l}+\mathbf{b}\right|^{2}=\frac{1}{2 A l^{2}}+\frac{1}{A l} b_{x}$.

The constant term is unimportant and we can omit it. Using the boundary condition (11), we obtain

$\hat{p}_{M}=-\frac{\mathrm{i}}{A l} \hat{\psi}$

that we insert in (13) to obtain the magnetic part of pressure stress

$\left\langle F_{p_{M}}\right\rangle=\frac{\left(\hat{\psi} \hat{\zeta}^{*}+\hat{\psi} * \hat{\zeta}\right)}{4 A l}$.

Combining (15) and (19) we find that the mean electromagnetic stress is zero as expected for an insulating solid region. The contribution from the nonlinear magnetic term is exactly zero because $b_{x}$ and $b_{z}$ are out of phase at $z=0$ [see (11) again]. The remaining fluid pressure stress is $\left\langle F_{p}\right\rangle-\left\langle F_{p_{M}}\right\rangle$.

When $\mathbf{B}_{0}$ is vertical, magnetic pressure is

$\hat{p}_{M}=\frac{1}{A l} \hat{\psi}$,

and we substitute again $\hat{p}$ with $\hat{p}_{M}$ in (13) to calculate the magnetic part of the pressure stress,

$\left\langle F_{p_{M}}\right\rangle=\frac{\mathrm{i}}{4 A l}\left(\hat{\psi} \hat{\zeta}^{*}-\hat{\psi}^{*} \hat{\zeta}\right)$

We verify that the magnetic pressure stress is neutralized on the solid side by the Lorentz term

$\left\langle F_{M, \mathcal{S}}\right\rangle=-\frac{1}{A l}\left\langle\left.\frac{\partial b_{x}}{\partial z}\right|_{z=0^{+}} \zeta\right\rangle$

which is once again obtained from linearizing the magnetic perturbation at the boundary.

\subsection{Mean forces in the fluid domain}

All forces in eq. (2) may contribute to the stress budget on the fluid side. Assuming there is no mass flux at infinity, the net stress associated with the nonlinear term $\mathbf{u} \cdot \nabla \mathbf{u}$ is zero:

$\mathbf{e}_{x} \cdot \int_{\mathcal{V}} \nabla \cdot(\mathbf{u} \otimes \mathbf{u}) \mathrm{d} \mathcal{V}=\mathbf{e}_{x} \cdot \int_{\mathcal{S}} \mathbf{u}(\mathbf{u} \cdot \mathbf{n}) \mathrm{d} \mathcal{S}=0$

as a result of the tensor divergence theorem and of the no-penetration condition $\mathbf{u} \cdot \mathbf{n}=0$ at the boundary. The linear part of the Reynolds stress $\mathbf{u} \cdot \nabla \mathbf{u}$ gives a contribution

$\left\langle-2 \frac{\mathrm{d} \zeta}{\mathrm{d} x} u_{x}+\frac{\partial u_{z}}{\partial z} \zeta\right\rangle=-\left\langle\frac{\mathrm{d} \zeta}{\mathrm{d} x} u_{x}\right\rangle$

which is exactly compensated by the nonlinear contribution

$\left\langle u_{x} u_{z}\right\rangle$

when the no-penetration condition (7) holds. Theoretically, it is not consistent to calculate the stress on the solid region only from the linear terms when keeping the Reynolds term in the modelling.

Any net flow in the $y$-direction produces a mean Coriolis force in the $x$-direction

$\frac{2}{R o} \iiint_{z<\zeta} u_{y} \mathrm{~d} V$. 
Table 1. Estimates of the various dimensional quantities in the Earth's core context and dimensionless numbers. Values adopted by Braginsky (1998), Glane \& Buffett (2018) and in this study (Section 4.4). Here, we consider the $\beta$-plane tangent to the surface of the core at $45^{\circ}$ latitude.

\begin{tabular}{lcccc}
\hline Quantity & Expression & Braginsky (1998) & Glane \& Buffett (2018) & This study \\
\hline Velocity & $U_{0}$ & $5 \times 10^{-4} \mathrm{~m} \mathrm{~s}^{-1}$ & $5 \times 10^{-4} \mathrm{~m} \mathrm{~s}^{-1}$ & $10^{-4} \mathrm{~m} \mathrm{~s}^{-1}$ \\
Vertical magnetic field & $B_{0}$ & $5 \times 10^{-4} \mathrm{~T}$ & $6.5 \times 10^{-4} \mathrm{~T}$ & $5 \times 10^{-4} \mathrm{~T}$ \\
Fluid density & $\rho_{0}$ & $10^{4} \mathrm{~kg} \mathrm{~m}^{-3}$ & $10^{4} \mathrm{~kg} \mathrm{~m}^{-3}$ & $10^{4} \mathrm{~kg} \mathrm{~m}^{-3}$ \\
Magnetic diffusivity & $\eta$ & $1 \mathrm{~m}^{2} \mathrm{~s}^{-1}$ & $0.8 \mathrm{~m}^{2} \mathrm{~s}^{-1}$ & $0.8 \mathrm{~m}^{2} \mathrm{~s}^{-1}$ \\
Pressure unit & $\rho_{0} U_{0}^{2}$ & $2.5 \times 10^{-3} \mathrm{~N} \mathrm{~m}^{-2}$ & $2.5 \times 10^{-3} \mathrm{~N} \mathrm{~m}^{-2}$ & $10^{-4} \mathrm{Nm}^{-2}$ \\
Unit length & $k_{x}^{-1}$ & $1.3 \times 10^{5} \mathrm{~m}$ & $1.6 \times 10^{4} \mathrm{~m}^{5}$ & $1.6 \times 10^{4}-1.3 \times 10^{5} \mathrm{~m}$ \\
Angular velocity & $\Omega$ & $5.16 \times 10^{-5} \mathrm{~s}^{-1}$ & $7.29 \times 10^{-5} \mathrm{~s}^{-1}$ & $5.16 \times 10^{-5} \mathrm{~s}^{-1}$ \\
Non-dimensional horizontal wavelength & $\chi_{x}$ & $7.4 \times 10^{-2}$ & - & $9.2 \times 10^{-3}-7.4 \times 10^{-2}$ \\
Alfvén number & $A l$ & $1.12 \times 10^{-1}$ & $8.6 \times 10^{-2}$ & $2.24 \times 10^{-2}$ \\
Rossby number & $R o$ & $7.6 \times 10^{-5}$ & $4.31 \times 10^{-4}$ & $1.52 \times 10^{-5}-1.22 \times 10^{-4}$ \\
Magnetic Reynolds number & $R_{m}$ & 64 & 10 & $2-16$ \\
Inverse Elsasser number & $Q$ & 2.59 & 1.72 & 2.06
\end{tabular}

Analogy between the planes $y=$ constant in our set-up and the cylindrical surfaces $s=$ constant in the sphere ( $s$ distance to the rotation axis) would require this term to be zero (Abdel-Aziz \& Jones 1988). This condition corresponds, in the sphere, to the Taylor's constraint (Taylor 1963). However, the zero net flow condition is not imposed in the linearized models discussed here and there is a net flow along the boundary in the direction orthogonal to its undulations. As a result, the Coriolis force acting on the fluid has non-zero mean. We find it to be

$\left\langle F_{C}\right\rangle=\frac{2}{R o}\left\langle\left. u_{y}\right|_{z=0} \zeta\right\rangle$

assuming that the undulations height is small compared to the vertical scale of the motions $\left(k_{z} \zeta \ll 1\right)$.

When $\mathbf{B}_{0}$ is horizontal, the Maxwell stress on the fluid is opposite to the Maxwell stress on the solid $\left\langle F_{M}\right\rangle$ and the total mean magnetic stress, expressed as $\left\langle F_{M}\right\rangle+\left\langle F_{p_{M}}\right\rangle$ on the solid, is zero on both sides. This is not true when $\mathbf{B}_{0}$ is vertical. There is a non-zero mean magnetic force in the fluid region,

$\left\langle F_{M, \mathcal{F}}\right\rangle=\frac{1}{A l}\left\langle\left.\frac{\partial b_{x}}{\partial z}\right|_{z=0^{-}} \zeta\right\rangle$

The discrepancy between the magnetic stress on the fluid and on the solid results from the discontinuity in the $x$-averaged $b_{x}$ at the fluid--solid interface, which gives rise to a current sheet in the $y$-direction. With $\mathbf{b}$ calculated at the order $\zeta$, the net electric current leaving the fluid region is $O\left(\zeta^{2}\right)$. Interaction between this current and the vertical magnetic field yields an $O\left(\zeta^{2}\right)$ magnetic force in the $x$-direction.

\subsection{Geophysical estimate of the mean stress at the core-mantle interface}

The axial torque exerted by the Earth's core on the mantle at decadal periods is about $10^{18} \mathrm{~N} \mathrm{~m}$, which corresponds to an average tangential stress of the order of $2.4 \times 10^{-3} \mathrm{~N} \mathrm{~m}^{-2}$ (Roberts \& Aurnou 2012). Glane \& Buffett (2018) and Moffatt (1977) adopted larger stresses of $2.7 \times 10^{-2}$ and $4 \times 10^{-2} \mathrm{~N} \mathrm{~m}^{-2}$ respectively as nominal values (i.e. axial torques of $1.1 \times 10^{19}$ and $1.6 \times 10^{19} \mathrm{Nm}$ ). This is appropriate if the friction torque cancels out most of the pressure torque as it occurs at the interface between the atmosphere and the solid Earth.

In order to evaluate models of tangential stress at the core-mantle interface, the first step is to calculate the pressure unit, which depends on the value of $U_{0}$. Moffatt (1977) assumed $U_{0}=10^{-4} \mathrm{~m} \mathrm{~s}^{-1}$, which is approximately the average zonal flow at the core surface. Braginsky (1998) and Glane \& Buffett (2018) supposed instead $U_{0}=5 \times 10^{-4} \mathrm{~m} \mathrm{~s}^{-1}$, which is a typical value of the rms surface velocity [see Gillet et al. (2015) for estimations of core surface velocities]. Since we seek to assess the average pressure stress in the azimuthal direction, the mean zonal flow appears to be the most appropriate estimate. The topography height $\zeta$ and the buoyancy frequency at the top of the Earth's core are very uncertain. Values for all other parameters of the problem are listed in Table 1.

\section{LineAR SOLUTION FOR AN IMPOSED MAGNETIC FIELD PARALLEL TO THE BOUNDARY}

Remarking that $R o \ll 1$ in the terrestrial context, Anufriev \& Braginskii (1975) and Moffatt (1977) neglected the inertia term in the momentum equation relative to the Coriolis term. When the magnetic field $B_{0} \mathbf{e}_{x}$ permeating the fluid region is strong enough $(Q \ll 1)$, an interior diffusion-free mode can be clearly distinguished from two boundary layer modes. Moffatt \& Dormy (2019, pp. 353-355) have recently discussed helicity generation (and the associated $\alpha$-effect) in the framework of this model. The configuration is sketched in Fig. 1. 


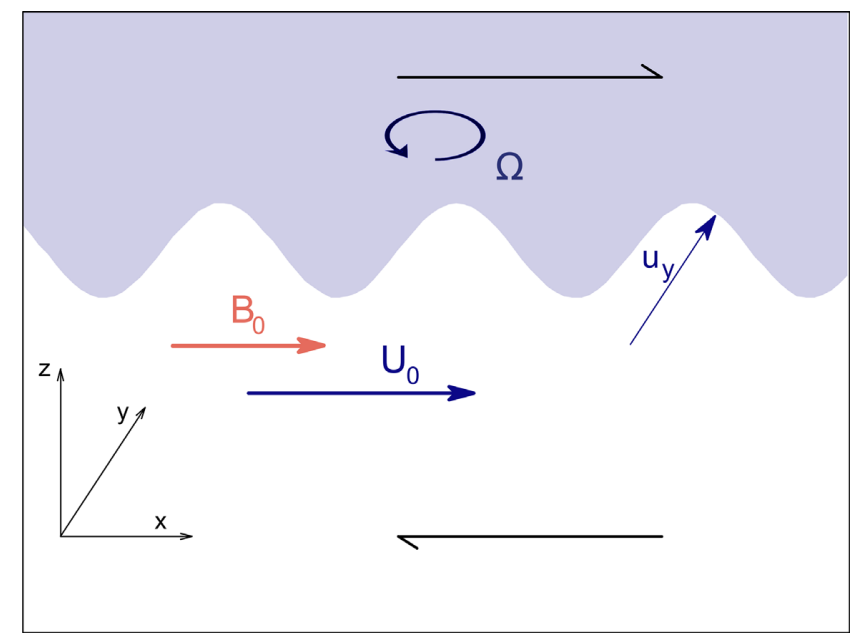

Figure 1. Sketch of the configuration investigated in Section 3. The vertical co-ordinate is stretched, and $k_{x} \hat{\zeta} \ll 1$ in the model. The solid (shaded region) is insulating. The fluid is conducting. The set-up is rotating and pervaded by a horizontal magnetic field $\mathbf{B}_{0}$. The fluid exerts a tangential pressure stress on the solid in the direction of the motion $U_{0}$. The pressure stress on the fluid is neutralized by the Coriolis forces arising from the $x$-averaged velocity along $\mathbf{e}_{y}$ in the valleys at the boundary.

\subsection{Linear modes}

From $\nabla \cdot \mathbf{b}=0$ and the $z$-component of the momentum equation, we have

$\hat{p}=-\frac{A l^{-1}}{k_{z}^{2}} \hat{b}_{x}$

for each mode. Then, the $x$ and $y$ components of the momentum equations are:

$$
\begin{aligned}
-2 \hat{u}_{y} & =\mathrm{i} R o A l^{-1} \frac{1+k_{z}^{2}}{k_{z}^{2}} \hat{b}_{x}, \\
2 \hat{u}_{x} & =\mathrm{i} R o A l^{-1} \hat{b}_{y} .
\end{aligned}
$$

Using $\nabla \cdot \mathbf{u}=0$, there are only two independent components of the induction equation:

$\mathrm{i} A l^{-1} \hat{u}_{x}-\mathrm{i} \hat{b}_{x}-R m^{-1}\left(1+k_{z}^{2}\right) \hat{b}_{x}=0$,

$\mathrm{i} A l^{-1} \hat{u}_{y}-\mathrm{i} \hat{b}_{y}-R m^{-1}\left(1+k_{z}^{2}\right) \hat{b}_{y}=0$.

It appears that there are solely two independent dimensionless numbers, $R_{m}^{-1}$ and $R o A l^{-2}$. Their ratio is the parameter $Q=A l^{2} / R o R_{m}$. Moffatt (1977) makes the assumption $Q \ll 1$. The system of eqs (30) and (31) has a non-trivial solution if its determinant vanishes:

$-Q^{-2}\left(1+k_{z}^{2}\right)=4 k_{z}^{2}\left(\mathrm{i} R_{m}+\left(1+k_{z}^{2}\right)\right)^{2}$

[same as eq. (36) of Moffatt \& Dillon (1976)]. When $R_{m} \gg 1, Q R_{m} \ll 1$, the three solutions for $k_{z}^{2}$ are approximately:

$k_{z}^{2}=-1, \quad k_{z}^{2}= \pm \frac{\mathrm{i}}{2 Q}$.

We are looking for solutions that vanish as $z \rightarrow-\infty$. Three solutions for $k_{z}$ satisfy this condition:

$k_{z, 1}=-\mathrm{i}, \quad k_{z, 2+}=\frac{1-\mathrm{i}}{2 \sqrt{Q}}, \quad k_{z, 2-}=-\frac{1+\mathrm{i}}{2 \sqrt{Q}}$.

The first root $k_{z, 1}$ makes the magnetic diffusion term vanish: it corresponds to the interior solution. Since $Q \ll 1$, the last two roots $k_{z, 2+}$ and $k_{z, 2-}$ satisfy $\left|k_{z}\right| \gg 1$ and correspond to a boundary layer solution, of vertical length scale $\sqrt{Q}$ [i.e. the parameter $\delta_{B}$ of Anufriev \& Braginskii (1975)] in the $z$-direction.

Appendix A gives expressions for the interior and boundary-layer modes. The boundary condition (9) involves both the interior mode $\mathbf{u}_{1}$ and the boundary-layer one $\mathbf{u}_{2}$. Inserting the expressions (A3) and (A5) for $u_{z, 1}$ and $u_{z, 2}$ into (9), we obtain the magnetic potential in the solid region as a function of topography

$\hat{\psi}=\frac{\mathrm{i} \hat{\zeta}}{A l\left(1-\frac{\mathrm{i}}{R_{m} \sqrt{Q}}\right)}$. 


\subsection{Vanishing net stress on the fluid}

The boundary layer contribution to the pressure is negligible relative to the interior contribution (29):

$\hat{p}=\frac{1}{A l} \hat{b}_{1, x}=\frac{\mathrm{i}}{A l} \hat{\psi}$.

Inserting (36) in expression (13) and combining it with (19), the traction exerted by the fluid pressure force on the solid domain is given by

$\left\langle F_{p}-F_{p_{M}}\right\rangle=-\frac{\left(\hat{\psi} \hat{\zeta}^{*}+\hat{\psi} * \hat{\zeta}\right)}{2 A l}$.

Finally, from expression (35) for the magnetic potential $\hat{\psi}$, we have

$\left\langle F_{p}-F_{p_{M}}\right\rangle=\frac{R_{m} \sqrt{Q}}{A l^{2}\left(1+R_{m}^{2} Q\right)}|\hat{\zeta}|^{2}=\frac{1}{R o \sqrt{Q}\left(1+R_{m}^{2} Q\right)}|\hat{\zeta}|^{2}$.

The corresponding expression in Moffatt (1977) is incorrect by a factor $3 / 2$ [see explanation after eq. (15) above].

The net flow in the corrugations produces a Coriolis force $\left\langle F_{C}\right\rangle$. Identity (27) transforms into

$\left\langle F_{C}\right\rangle=\frac{1}{2 R o}\left(\hat{u}_{y} \hat{\zeta}^{*}+\hat{u}_{y}^{*} \hat{\zeta}\right)=-\frac{\left(\hat{\psi} \hat{\zeta}^{*}+\hat{\psi} * \hat{\zeta}\right)}{2 A l}$,

where $\hat{u}_{y}$ is obtained from (A5). Noting that (39) gives the mean Coriolis stress on the fluid domain whereas (37) is for the solid region, we verify that the net stress on the fluid side is zero, as it should.

\subsection{Electrical current sheet}

We can calculate the electric current density $j_{y}$ next to the interface,

$j_{y}=-\frac{\mathrm{i} \hat{\psi}}{\sqrt{Q}} \exp (\mathrm{i} x), \quad$ at $\quad z=0$

from the expression of $b_{2, x}$ given in Appendix A (with $\sqrt{\rho / \mu} U_{0} k_{x}$ chosen as unit of electric current density). These currents are not out of phase with the topography and hence there is a net flux of electrical currents along the boundary, in the corrugations:

$\overline{J_{y}^{\zeta}}=-<\frac{\mathrm{i} \psi}{\sqrt{Q}} \zeta>=\frac{R_{m}^{3 / 2}}{2 \sqrt{R o}\left(1+R_{m}^{2} Q\right)}|\zeta|^{2}$,

where the overline notation means that the quantity is $x$-invariant. This surface current flux yields a jump in $\bar{b}_{x}$ across the surface layer, $z=$ 0 . We have

$\left.\overline{b_{x}}\right|_{z=0^{-}}=-\overline{J_{y}^{\zeta}}$

since $\left.\overline{b_{x}}\right|_{z=0^{+}}=0$. This field opposes the base magnetic field $\mathbf{B}_{\mathbf{0}}$. Similarly, there is a net flux of electrical currents in the $x$ direction, $\overline{J_{x}^{\zeta}}=\overline{J_{y}^{\zeta}}$.

\subsection{Geophysical application}

Multiplying by the unit of stress $\rho U_{0}^{2}$, we remark that $\left\langle F_{p}-F_{p_{M}}\right\rangle$ is a linear function of $U_{0}$ when $R_{m}^{2} Q \ll 1$. We can verify also that it varies inversely with the topography wavelength. For an horizontal imposed magnetic field of $3 \times 10^{-3} \mathrm{~T}$, a velocity $U_{0}$ of $10^{-4} \mathrm{~m} \mathrm{~s}^{-1}$ and a topography wavelength of $800 \mathrm{~km}$, we find a pressure stress of $2 \times 10^{-3} \mathrm{~N} \mathrm{~m}^{-2}$, which is of the order of the total mean tangential stress at the core-mantle interface (see Section 2.4).

\section{LinEAR SOLUTION FOR A STRATIFIED FLUID PERMEATED BY A MAGNETIC FIELD TRANSVERSE TO THE BOUNDARY}

Braginsky (1998) and Glane \& Buffett (2018) included fluid stratification in their models. The imposed magnetic field acts in the $z$-direction $\left(\mathbf{e}_{B}=\mathbf{e}_{z}\right)$ and the Eulerian advection term $\partial \mathbf{u} / \partial x$ is retained in the momentum equation. Braginsky (1998) further assumed quasi-geostrophy. His study is based on the $\beta$-plane vorticity equation. Glane \& Buffett (2018) focused instead on short wavelength topography, for which effects due to the sphericity of the Earth can be ignored. Then, eqs (2)-(4) are appropriate to model the dynamics in the plane layer tangent to the core-mantle boundary ( $f$-plane approximation). I present the two models of Braginsky (1998) and Glane \& Buffett (2018) as asymptotic limits of a general model that includes both variation in latitude of the radial component of the rotation vector ( $\beta$-plane approximation) and wide range of imposed magnetic field strength. The problem is depicted in Fig. 2. 


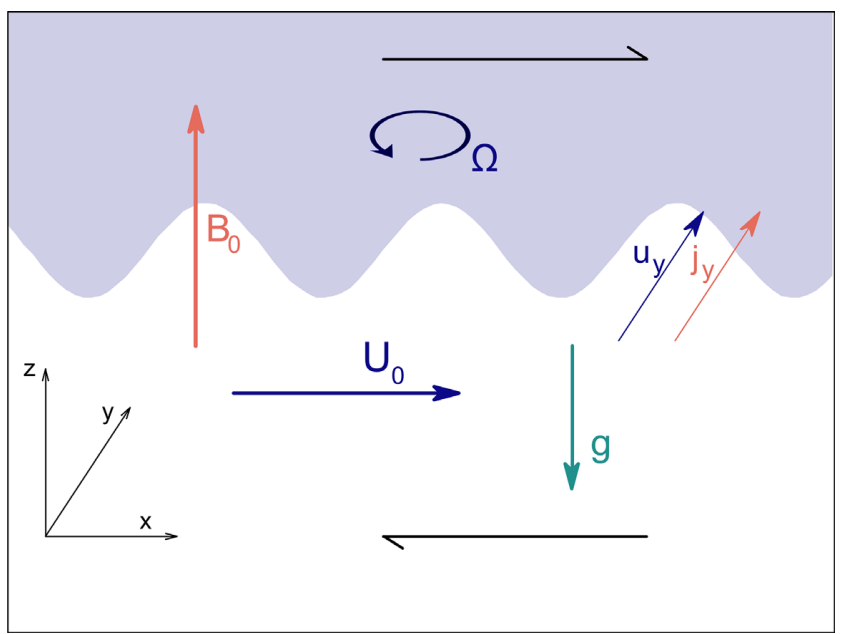

Figure 2. Sketch of the configuration investigated in Section 4. The solid (shaded region) is insulating. The fluid is conducting and stably stratified. The set-up is rotating. A uniform vertical magnetic field $\mathbf{B}_{0}$ is imposed. The fluid exerts a tangential pressure stress on the solid in the direction of the motion $U_{0}$. The pressure stress on the fluid (in the opposite direction) is neutralized by the magnetic and Coriolis forces arising from the $x$-averaged current and velocity along $\mathbf{e}_{y}$ in the valleys at the boundary.

\subsection{Linear modes}

The fluid layer is stably stratified,

$\bar{\rho}=\rho_{0}(1+\alpha z)$,

where $\alpha$ is constant and related to the buoyancy frequency $N\left(\alpha=-N^{2} / g\right)$. Vertical transport produces density anomalies, as evidenced by the mass conservation equation,

$\frac{\partial \rho^{\prime}}{\partial x}+u_{z} \frac{\mathrm{d} \bar{\rho}}{\mathrm{d} z}=0$.

The momentum eq. (2) is modified by the introduction of a buoyancy term

$\mathrm{iu}+\left(2+\chi_{x} y\right) R o^{-1} \mathbf{e}_{z} \times \mathbf{u}=-\nabla p+A l^{-1} \mathbf{e}_{z} \cdot \nabla \mathbf{b}+\mathrm{i} \frac{u_{z}}{F r^{2}} \mathbf{e}_{z}$,

where the last term stands for $-\rho^{\prime} g$ in dimensionless form and the Froude number $F r$ is defined from $F r=\left|k_{x} U_{0} / N\right|$. As in Braginsky (1998), we assume here the effective rotation vector to be $2 \Omega+\beta y$ ( $\beta$-plane approximation with y standing for latitude) and $\chi_{x}=\beta / k_{x} \Omega$ arises as a measure of the horizontal wavelength of the topography.

I assume henceforth strong stratification $F r \ll 1$, which yields the hydrostatic equilibrium approximation. Then, the $z$-component of (45) gives

$\hat{p}=-\frac{1}{k_{z}^{2} F r^{2}} \hat{u}_{x}$.

The variation of $\Omega$ with $y$ appears in the vorticity equation

$-u_{y}+2 \mathrm{i} R o^{-1} u_{x}+\chi_{x} R o^{-1} u_{y}=-k_{z} A l^{-1} b_{y}$,

where the pressure term is eliminated. We complement this equation with the $x$-component of the momentum equation (45), where we consider $\Omega$ uniform.

We can now transform the induction equation (3), the $x$-component of (45) and the vorticity equation (47) into a linear system of four equations for $\hat{u}_{x}, \hat{u}_{y}, \hat{b}_{x}, \hat{b}_{y}$ of which the coefficients are function of $k_{z}$. There is a non-trivial solution if the determinant vanishes,

$F r^{2} k_{z}^{6}+A l^{2} k_{z}^{2}\left(1+\left(\frac{\chi_{x}}{R o}-2\right) k_{z}^{2} F r^{2}\right)\left(\gamma^{2}-\mathrm{i} \frac{k_{z}^{2}}{R_{m}}\right)=A l^{4}\left(1-\frac{\chi_{x}}{R o}+4 \frac{k_{z}^{2} F r^{2}}{R o^{2}}\right)\left(\gamma^{2}-\mathrm{i} \frac{k_{z}^{2}}{R_{m}}\right)^{2}$,

where we have used $R o \ll 1$ and $\gamma$ is the square root of $\left(1-\mathrm{i} R_{m}^{-1}\right)$ with negative imaginary part:

$\gamma^{2}=1-\frac{\mathrm{i}}{R_{m}}, \quad \Im(\gamma)<0$. 
We estimate the three solutions of the cubic equation (48) for $k_{z}^{2}$ assuming once again $F r \ll 1$ together with $R o \ll 1$ and $F r^{2} \ll R o Q$. We obtain

$k_{z, 1}^{2}=\gamma^{2} R o R_{m} Q\left(1-\frac{\chi_{x}}{R o}\right), \quad k_{z, 2}^{2}=-\mathrm{i} \gamma^{2} R_{m}+\mathrm{i} \gamma^{4} \frac{R_{m}^{2} F r^{2}}{Q R o}$,

$k_{z, 3}^{2}=\mathrm{i} \frac{\operatorname{Ro} Q}{F r^{2}} \frac{1-\mathrm{i} Q \chi_{x}}{1-\mathrm{i} Q \chi_{x}+4 Q^{2}}$.

Expressions for the three modes are derived in Appendix B.

\subsection{Horizontal stress balance}

We gather in Appendix B that it is sufficient to consider the third mode, which is the boundary layer one, to deduce the dominant terms in the pressure stress on the solid in the limit $F r^{2} \ll R o Q$. Under the same condition, the magnetic pressure stress $\left\langle F_{p M}\right\rangle$ is negligible relative to $\left\langle F_{p}\right\rangle$. Eq. (B23) of Appendix B gives the pressure stress as a function of the dimensionless numbers. We can express it as

$\left\langle F_{p}\right\rangle=-\frac{1}{2 F r \sqrt{\operatorname{RoQ}}}\left(\frac{\left(1+4 Q^{2}\right)^{2}+\left(Q \chi_{x}\right)^{2}}{1+\left(Q \chi_{x}\right)^{2}}\right)^{1 / 4} \frac{\Re\left(k_{z, 3}\right)}{\left|k_{z, 3}\right|}|\hat{\zeta}|^{2}$

[see eq. (B17) for the expression of $\left.\Re\left(k_{z, 3}\right) /\left|k_{z, 3}\right|\right]$. We have

$\frac{\Re\left(k_{z, 3}\right)}{\left|k_{z, 3}\right|}=-\frac{1}{\sqrt{2}}$

when $\chi_{x}=0$. As observed by Braginsky (1998) and Glane \& Buffett (2018), $\left\langle F_{p}\right\rangle$ increases linearly with $F r^{-1}$, i.e. with the buoyancy frequency $N$. Since $Q$ and $\chi_{x}$ are independent of $U_{0}$, the dimensionless pressure stress $\left\langle F_{p}\right\rangle$ scales as $U_{0}^{-3 / 2}$. Multiplying by the unit of stress $\rho U_{0}^{2}$, we find that the mean pressure stress varies with velocity as $\sqrt{U_{0}}$ [see eq. (57 b) of Braginsky (1998) and fig. 4C of Glane \& Buffett (2018)].

On the fluid side, the Reynolds stress term is negligible provided $R o Q \ll 1$ (see again Appendix B). The pressure stress $-\left\langle F_{p}\right\rangle$ has to be compensated by the mean Coriolis and magnetic forces, $\left\langle F_{C}\right\rangle$ and $\left\langle F_{M, \mathcal{F}}\right\rangle$ since there is no acceleration. In Appendix $\mathrm{B}$, $\left\langle F_{C}\right\rangle+\left\langle F_{M, \mathcal{F}}\right\rangle-\left\langle F_{p}\right\rangle=0$ we verify in the general case. To make the discussion simpler, we examine here the case $Q \chi_{x} \equiv 0$. Then, we have

$\frac{\left\langle F_{C}\right\rangle}{\left\langle F_{p}\right\rangle}=\frac{4 Q^{2}}{1+4 Q^{2}}, \quad \frac{\left\langle F_{M, \mathcal{F}}\right\rangle}{\left\langle F_{p}\right\rangle}=\frac{1}{1+4 Q^{2}}$.

When $Q \gg 1$, the pressure stress on the fluid is mostly neutralized by the Coriolis force, as in the homogeneous model with horizontal magnetic field (see Section 3). When $Q \ll 1$, it is balanced by the magnetic force instead. This force has no counterpart in the solid, which is electrically insulating. It is necessary to consider $O\left(\zeta^{2}\right)$ perturbations to cancel out both the mean Coriolis force and the mean magnetic force in the $x$-direction.

\subsection{Asymptotic limits: quasi-geostrophy versus small horizontal length scale}

We recover the models of Braginsky (1998) and Glane \& Buffett (2018) as limit cases.

The solution of Braginsky (1998) can be obtained as the quasi-geostrophic limit $(Q \gg 1)$ of the solution presented above. In this limit, we have

$k_{z, 3}^{2}=\frac{R o}{4 F r^{2} Q}\left(i+Q \chi_{x}\right)$

Braginsky referred to the mode of wavenumber $k_{z, 3}$ as the velocity mode and to the mode of wavenumber $k_{z, 1}$ as the magnetic mode. The original model of Braginsky slightly differs from the model described here inasmuch it includes a density jump between the stratified layer and the interior far from the boundary. However, this difference is unimportant since Braginsky noted that the mean pressure stress, in his model, mostly results from the boundary layer mode, which is not affected by the composition change deep inside the fluid. From (54), we verify $k_{x}^{2} k_{z, 3}^{2}=\left(-\mathrm{i} \kappa_{v}\right)^{2}$, where $\left(-\mathrm{i} \kappa_{v}\right)$ is Braginsky's notation for the vertical wavenumber (in $\mathrm{m}^{-1}$ ) of the boundary layer mode. Therefore, the two models also agree about the mean pressure stress, which depends only on $k_{z, 3}$ (see eq. B23). We have

$$
\begin{aligned}
\left\langle F_{p}\right\rangle & =-\frac{\sqrt{Q}}{F r \sqrt{R o}\left(1+\left(Q \chi_{x}\right)^{2}\right)^{1 / 4}} \frac{\Re\left(k_{z, 3}\right)}{\left|k_{z, 3}\right|}|\hat{\zeta}|^{2}, \\
\frac{\Re\left(k_{z, 3}\right)}{\left|k_{z, 3}\right|} & =-\frac{1}{\sqrt{2}} \sqrt{1+\frac{Q \chi_{x}}{\left(1+\left(Q \chi_{x}\right)^{2}\right)^{1 / 2}}}, \quad k_{z, 3}=\left|k_{z, 3}\right| \exp \left(\mathrm{i} \varphi_{0}\right),
\end{aligned}
$$

where $\varphi_{0}$ changes from $5 \pi / 4$ to $\pi$ as $Q \chi_{x}$ goes from 0 to $\infty$. The factor 2 discrepancy with the expression (57a) of Braginsky (1998) arises from the description of the topography as a 2-D function in his article. 

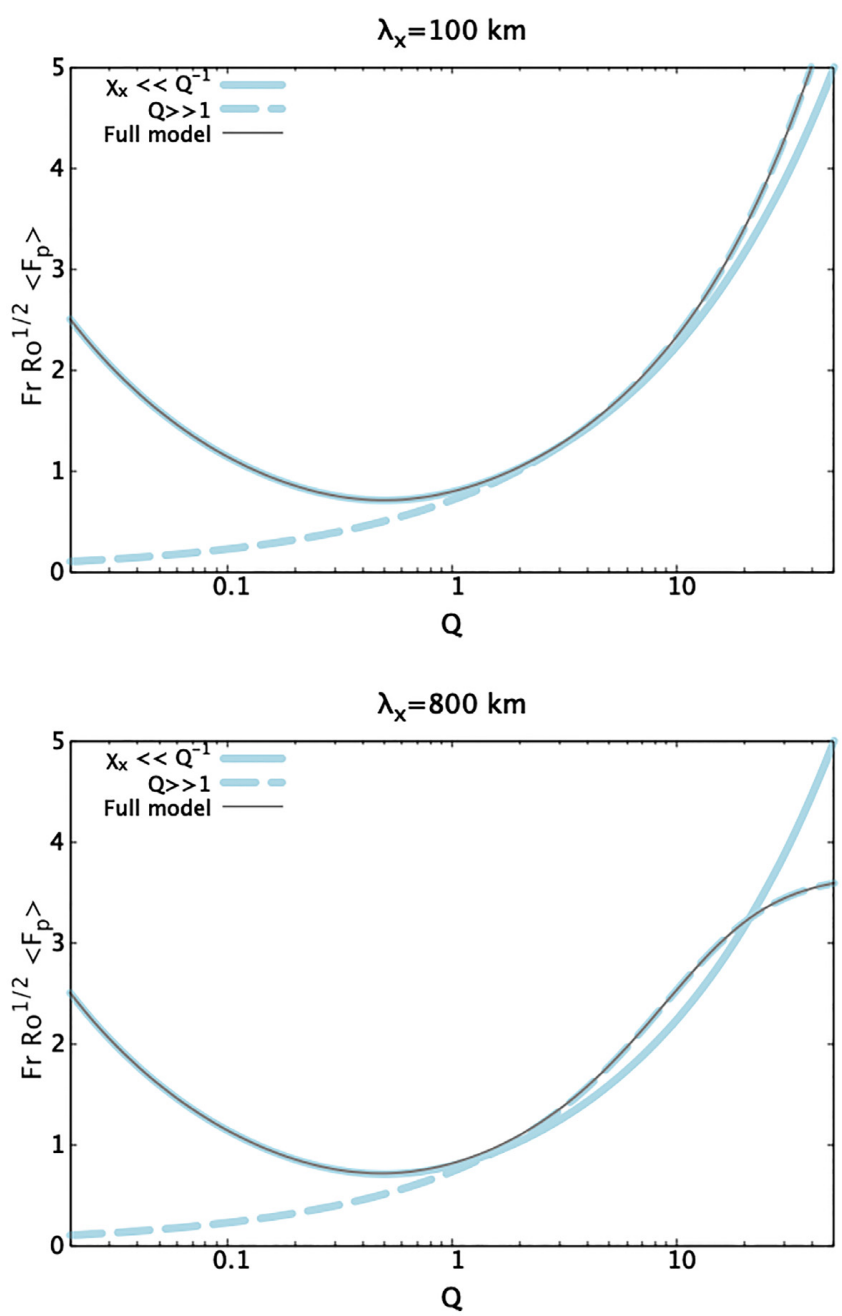

Figure 3. Tangential stress as a function of $Q$ (thin black line). Comparison with the short wavelength approximation $\chi_{x} \ll Q^{-1}$ of Glane \& Buffett (2018) (thick full blue line) and the quasi-geostrophic approximation $Q \gg 1$ of Braginsky (1998) (thick dashed blue line) for two horizontal wavelengths of the topography, $100 \mathrm{~km}$ (top) and $800 \mathrm{~km}$ (bottom). All models agree for $Q \sim 2$.

The model of Glane \& Buffett (2018) corresponds to the limit of small horizontal wavelength $Q \chi_{x} \ll 1$. In this limit, we have

$k_{z, 3}=-\frac{1+\mathrm{i}}{\sqrt{2}} \frac{\sqrt{\operatorname{RoQ} Q}}{F r} \frac{1}{\sqrt{1+4 Q^{2}}}$.

Replacing the dimensionless numbers by the nominal set of Glane \& Buffett (listed in Table 1), together with $\chi_{x}=0$, and adopting their value for the wavenumber unit, we recover solution (11) of their paper, which they obtained after substituting numerical values in algebraic expressions. As a matter of fact, an expression equivalent to (56) for $k_{z, 3}$ can be found in the original unpublished version of their article (B. Buffett, personal communication, 2019).

Fig. 3 shows the quantity $\operatorname{Fr} \sqrt{\operatorname{Ro}}\left\langle F_{p}\right\rangle$ for the two values of $k_{x}$, adopted by Glane \& Buffett (2018) and Braginsky (1998) respectively. Their models can be presented as special cases of the complete model. They coincide in the parameter range

$1 \ll Q \ll \chi_{x}^{-1}$.

They extend respectively to strong magnetic fields $(Q<1)$ and to large topography wavelengths $\left(\chi_{x}>Q^{-1}\right)$. Both models are applicable for $Q \sim 2$, which is the geophysically relevant value (see Table 1 ).

\subsection{Minimum topography height for the model to be applicable}

Although the pressure stress increases linearly with buoyancy frequency $N$, there is a restriction on the range of $N$ values to be considered since the thickness $\delta_{B}$ of the boundary layer varies inversely with $N$. The perturbation approach requires that the bumps are well within the 
boundary layer

$|\zeta| \ll \delta_{B}, \quad$ with $\quad \delta_{B}=\left(\Im\left(k_{z, 3}\right)\right)^{-1}=\sqrt{2} \frac{\sqrt{1+4 Q^{2}} F r}{\sqrt{R o Q}}$,

which is equivalent to

$\operatorname{Fr} \gg \frac{1}{\sqrt{2}} \frac{\sqrt{R o Q}}{\sqrt{1+4 Q^{2}}}|\zeta|$,

assuming here $Q \chi_{x} \equiv 0$. This sets an upper limit on the stratification to ensure that the perturbation approach is justifiable. Using (7) and (4, right), this condition amounts to $u_{x} \ll 1$, which is also a prerequisite for linearization. Inserting the result (59) in the expression of the pressure stress (51), we obtain a condition on the pressure stress that depends linearly on the topography amplitude $|\zeta|$ :

$\left\langle F_{p}\right\rangle \ll \frac{1+4 Q^{2}}{2 \operatorname{Ro} Q}|\zeta|$.

The maximizing function attains its minimum for $Q=0.5$. Multiplying by the unit of stress $\rho U_{0}^{2}$, we obtain $2 \rho \Omega U_{0} \zeta^{*}$ as minimum value of this function (where $\zeta^{*}$ dimensional value of $|\zeta|$ ). Interestingly, this nearly corresponds to the reference value for the topographic stress obtained from dimensional analysis (Hide 1977). In the limit $Q \ll 1$, the restriction on the pressure stress transforms into

$\left\langle F_{p}\right\rangle \ll \frac{1}{2} \sigma \zeta^{*} B_{0}^{2} U_{0}$,

which compares nicely with the electromagnetic stress $\sigma \zeta^{*} B_{0}^{2} U_{0}$ on an electrically conducting solid with conductance $\sigma \zeta^{*}(\sigma$ electrical conductivity of the fluid). For $Q \sim 1$ or larger, the analogy does not hold.

Using $2.4 \times 10^{-3} \mathrm{~N} \mathrm{~m}^{-2}$ as target value of the tangential stress $\left\langle F_{p}\right\rangle$ and $100 \mathrm{~km}$ as the topography wavelength, restriction (60) on $\left\langle F_{p}\right\rangle$ translates into $\zeta^{*} \gg 11 \mathrm{~m}$. To give an example of a possible set of values, $\zeta^{*}=100 \mathrm{~m}$ corresponds to $F r=1.55 \times 10^{-4}$ (i.e. $N / \Omega=0.8$ ) and to a boundary layer thickness $\delta_{B}$ of $930 \mathrm{~m}$. The larger value adopted by Glane \& Buffett (2018) as nominal tangential stress leads to $\zeta^{*}$ $\gg 60 \mathrm{~m}$. The difference with the topography amplitude given in their article originates in the value chosen for $U_{0}$.

\subsection{Physical interpretation in the large magnetic field limit}

Glane \& Buffett (2018) posited that stratification traps the fluid next to the solid boundary in topography elevations. According to this scenario, the perturbation $u_{x}$ would oppose $U_{0}$ within the bumps. Further, they suggested that the coupling mechanism is similar to electromagnetic coupling between the fluid in the interior and stagnant fluid within topography rises, as initially advocated to explain nutation observations (Buffett 2010).

When $Q \ll 1$ (large magnetic field limit) and $Q \chi_{x} \equiv 0$, expression (61) shows that the magnetic coupling interpretation gives a good prediction of the tangential stress (within a factor $1 / 2$ ) for boundary layer modes of vertical scale $\delta_{B}$ similar to the topography height $|\zeta|$. The amplitude of $u_{x}$ becomes comparable to 1 (i.e. $U_{0}$ in physical units) precisely for this value of $\delta_{B}$. The velocity perturbation would exactly oppose $U_{0}$ within the bumps for a phase difference $\pi$ between $u_{x}$ and $\zeta$. For $Q \chi_{x} \equiv 0$, the phase difference reaches $3 \pi / 4$ (see eq. 52).

This interpretation rests on an extrapolation of the solution outside its domain of validity (topography height and flow perturbation amplitude small compared to the boundary layer depth and the base flow velocity, respectively). Another study including nonlinearities is needed to calculate the solution for large undulations $|\zeta| \sim \delta_{B}$ and prove or disprove, at least in the small $Q$ limit, the interpretation put forward by Buffett (2010) and Glane \& Buffett (2018). This goes beyond the scope of this paper.

\section{TRANSFER OF MOMENTUM FROM THE BOUNDARY INTO THE FLUID}

There are two forces, with non-zero averages, acting on the fluid side in addition to the fluid pressure stress $\left\langle-F_{p}+F_{p M}\right\rangle$ : the magnetic stress $\left\langle-F_{M}-F_{p M}\right\rangle$, also written as $\left\langle F_{M, \mathcal{F}}\right\rangle$, and the Coriolis force $F_{C}$. These two terms do not represent interactions with the solid region. They arise instead from flux of electrical currents and of mass at infinity, which do not occur in closed bodies. When the imposed magnetic field $\mathbf{B}_{0}$ permeating the set-up is horizontal, the tangential stresses are balanced only by the Coriolis force $F_{C}$ due to the mean volume flux since there is no magnetic force arising from the mean electrical currents. When $\mathbf{B}_{0}$ is vertical, both the Coriolis force and the magnetic force participate in the equilibrium. Then, the Coriolis and magnetic forces predominate for large and small inverse Elsasser number $Q$ respectively. In both cases, the success of the models hinges on finite net electrical current and volume fluxes leaving the fluid region. They yield non-zero mean electromagnetic and Coriolis forces on the fluid that are balanced not in the solid region but at infinity. This feature is undesirable as there can be no such fluxes in the geophysical situation.

I discuss here the $O\left(\zeta^{2}\right) x$-averaged time-varying fields when the imposed magnetic field is parallel to the boundary. The study when the magnetic field is transverse and the fluid is stratified is much more difficult.

We are guided by studies of viscous friction in rotating flows. Viscous stresses can be estimated from Ekman layer models although these models do not necessarily include the acceleration $\partial \mathbf{u} / \partial t$. Therefore, study of the viscous boundary layer is completed by investigation of the fluid response in the interior driven by Ekman pumping. 
After $x$-averaging, the 2-D problems discussed in Sections 3 and 4 become 1-D. In order to avoid the indeterminacy typical to 1-D problems and noted, for example, by Jackson (1975, p. 477) in a related context (Hartmann boundary layer in plane layer geometry), I consider in this section a 2-D topography:

$\zeta(x, y)=\Re\left(\hat{\zeta}\left(\exp \left(\mathrm{i}\left(x+k_{y} y\right)\right)+\exp \left(\mathrm{i}\left(x-k_{y} y\right)\right)\right)\right)$,

defined in such a way that, after $x$-averaging, there remains a $y$-dependent component in the tangential stresses. Also, I find it advantageous to introduce the characteristic length $k_{y}^{-1}$ because the group velocity of magnetic Coriolis waves spawn in the fluid interior from the boundary depends on the horizontal wave length [see Greenspan (1968, pp. 192-195) and Davidson (2014) for the discussion of inertial waves propagation].

The $x$-averaged traction on the topography (62) is calculated in Appendix C, where I first calculate the linear solution for each Fourier component. Keeping the same hypotheses as in Section 3, the two wavenumbers $k_{z, 2+}$ and $k_{z, 2-}$ are unchanged while $k_{z, 1}$ becomes

$k_{z, 1}=-\mathrm{i} k_{\|}, \quad$ with $\quad k_{\|}=\sqrt{1+k_{y}^{2}}$.

Expression (C7) for the $x$-averaged traction on the solid involves both $k_{y}$ and the horizontal wavenumber $k_{11}$. It is obtained as the sum of a constant and of a sinusoidal components. I discuss here only the sinusoidal one since it corresponds to a well-posed problem.

The net flux of electrical current and mass along the interface have to be balanced by opposite flux in the fluid volume, which drive 2-D perturbations. Because the different fields are independent of $x$, the momentum and induction equations for these perturbations are now decoupled. In the absence of uniform pressure gradient and mean electrical field, we have to keep acceleration terms $\partial \mathbf{u} / \partial t$ and $\partial \mathbf{b} / \partial t$ to balance the equations. Inertial velocity modes and magnetic diffusion modes are solution of the momentum and induction equation respectively.

The flux of electrical current $\overline{J_{\zeta}}$ along the interface in either direction $x$ or $y$ is a function of $\exp \left(2 \mathrm{i} k_{y} y\right)$. It gives a boundary condition for the magnetic field in the fluid volume at $z=0$ (see e.g. eq. 42) for $\overline{b_{x}}$ ). The magnetic field obeys a diffusion equation

$\frac{\partial \overline{b_{x}}}{\partial t}=R_{m}^{-1} \nabla^{2} \overline{b_{x}}$

with boundary conditions:

$\left.\overline{b_{x}}\right|_{z=0}=-\overline{J_{y}^{\zeta}},\left.\quad \overline{b_{x}}\right|_{z=-\infty}=0$.

Then the flux of electrical currents in the fluid volume exactly compensates for the flux along the interface.

By analogy with the relationship between electrical currents and magnetic fields, the velocity field can be written as

$\mathbf{u}=\left(w, \frac{\partial v}{\partial z},-\frac{\partial v}{\partial y}\right)$

Writing

$\left.v\right|_{z=0}=-\left\langle u_{y} \zeta\right\rangle, \quad v_{z=-\infty}=0$

where $\left\langle u_{y} \zeta\right\rangle$ is the mass flux along the interface ensures that the mass flux in the fluid volume and along the interface compensate each other. It corresponds to the boundary condition

$u_{z}=\frac{\partial}{\partial y}\left\langle u_{y} \zeta\right\rangle$

for the velocity field. The solutions consist of inertial waves forced at $z=0$. Denoting $\omega$ the frequency of plane waves of the form

$p=\Re\left(\hat{p} \exp \left(\mathrm{i}\left(\omega t+k_{y} y+k_{z} z\right)\right)\right)$,

with similar expressions for the velocity components, we obtain the dispersion relation

$\omega= \pm \frac{2}{R o} \sqrt{\frac{k_{z}^{2}}{k_{y}^{2}+k_{z}^{2}}}$.

As the waves propagate along the vertical axis (with $k_{z} \ll k_{y}$ ), they make the motions $z$-invariant (Davidson 2014). Thus, they are the mechanism to transfer momentum from the boundary to the fluid interior.

\section{CONCLUDING REMARKS}

In this paper, the tangential stress results from a steady flow over boundary topography. The two mechanisms that have been proposed to confine the perturbations close to the fluid/solid interface are evaluated. The first mechanism hinges on the presence of a strong magnetic field parallel to the interface. Then, the perturbations are localized next to the interface provided that $Q \ll 1$, where $Q$ is the inverse Elsasser number (see Section 3). The other mechanism requires strong stratification to confine the perturbations next to the interface (Section 4 ). In 
both cases, the pressure stress acting on the fluid half-space in the direction parallel to $U_{0}$ is balanced by the magnetic and Coriolis forces arising from the net flux of electrical currents and of mass along the interface.

The tangential stress calculation leaves aside the question of momentum transfer to the interior. When the fluid is not stratified and the imposed magnetic field horizontal, inertial waves make the fluid response $z$-invariant and ensure efficient momentum transfer from the boundary to the interior.

There remains a lot to be done about the tangential pressure stress at the boundary between a stratified core and a solid mantle. We have to investigate whether the modes forced at the boundary are evanescent and dissipated over a short distance or are able to propagate as the inertial waves discussed in Section 5. Furthermore, the linear models discussed in this article cannot describe the situation where the boundary layer thickness is of the order of the height of the topography. The possible analogy with electromagnetic coupling, albeit not justified for linearized models, makes this configuration very interesting.

Finally, similarly to the gravity wave torque in the atmosphere, the traction exerted by the pressure on the boundary needs to be considered together with other stress terms.

\section{ACKNOWLEDGEMENTS}

This work has been carried out with financial support from CNES (Centre National d'Études Spatiales, France).

\section{REFERENCES}

Abdel-Aziz, M.M. \& Jones, C.A., 1988. $\alpha \omega$-dynamos and Taylor's constraint, Geophys. astrophys. Fluid Dyn., 44, 117-139.

Amit, H. \& Pais, M.A., 2013. Differences between tangential geostrophy and columnar flow, Geophys. J. Int., 194, 145-157.

Anufriev, A.P. \& Braginskii, S.I., 1975. Effect of a magnetic field on the stream of liquid rotating at a rough surface, Magnetohydrodynamics, 11, 461-467.

Bärenzung, J., Holschneider, M., Wicht, J., Sanchez, S. \& Lesur, V., 2018. Modeling and predicting the short-term evolution of the geomagnetic field, J. geophys. Res., 123, 4539-4560.

Braginsky, S.I., 1998. Magnetic Rossby waves in the stratified ocean of the core, and topographic core-mantle coupling, Earth Planets Space, 50, 641-649.

Buffett, B.A., 2010. Chemical stratification at the top of Earth's core: constraints from observations of nutations, Earth planet. Sci. Lett., 296, 367372.

Davidson, P., 2014. The dynamics and scaling laws of planetary dynamos driven by inertial waves, Geophys. J. Int., 198, 1832-1847.

Egger, J., Weickmann, K. \& Hoinka, K.-P., 2007. Angular momentum in the global atmospheric circulation, Rev. Geophys., 45(RG4007), doi:10.1029/2006RG000213.

Gillet, N., Jault, D. \& Finlay, C.C., 2015. Planetary gyre, time-dependent eddies, torsional waves, and equatorial jets at the Earth's core surface, $J$. geophys. Res., 120, 3991-4013.

Glane, S. \& Buffett, B., 2018. Enhanced core-mantle coupling due to stratification at the top of the core, Front. Earth Sci., 6, 171, doi:10.3389/feart.2018.00171.

Greenspan, H.P., 1968. The Theory of Rotating Fluids, Cambridge Univ. Press.

Hassan, M.H.A. \& Eltayeb, I.A., 1982. On the topographic coupling at the core-mantle interface, Phys. Earth planet. Inter., 28, 14-26.

Helffrich, G. \& Kaneshima, S., 2010. Outer-core compositional stratification from observed core wave speed profiles, Nature, 468, 807-812.

Hide, R., 1977. Towards a theory of irregular variations in the length of the day and core-mantle coupling, Phil. Trans. R. Soc. Lond., A, 284, 547-554.

Hide, R., 1989. Fluctuations in the Earth's rotation and the topography of the core-mantle interface, Phil. Trans. R. Soc. Lond., A, 328, 351-363.
Irving, J.C.E., Cottaar, S. \& Lekić, V., 2018. Seismically determined elastic parameters for Earth's outer core, Sci. Adv., 4(6), eaar2538, doi:10.1126/sciadv.aar2538.

Jackson, J.D., 1975. Classical Electrodynamics, John Wiley \& Sons.

Jault, D. \& Le Mouël, J.L., 1990. Core-mantle boundary shape: constraints inferred from the pressure torque acting between the core and the mantle, Geophys. J. Int., 101, 233-242.

Jault, D., Gire, C. \& Le Mouël, J.L., 1988. Westward drift, core motions and exchanges of angular momentum between core and mantle, Nature, 333, 353-356.

Kuang, W. \& Chao, B.F., 2001. Topographic core-mantle coupling in geodynamo modeling, Geophys. Res. Lett., 28, 1871-1874.

Lambeck, K., 1980. The Earth's Variable Rotation. Geophysical Causes and Consequences, Cambridge Univ. Press.

McIntyre, M.E., 1980. An introduction to the generalized Lagrangian-mean description of wave, mean-flow interaction, Pure appl. Geophys., 118, 152-176.

Moffatt, H.K., 1977. Topographic coupling at the core-mantle interface, Geophys. astrophys. Fluid Dyn., 9, 279-288.

Moffatt, H.K. \& Dillon, R.F., 1976. The correlation between gravitational and geomagnetic fields caused by interaction of the core fluid motion with a bumpy core-mantle interface, Phys. Earth planet. Inter., 13, 67-78.

Moffatt, K. \& Dormy, E., 2019. Self-exciting Fluid Dynamos, Cambridge Univ. Press.

Pais, M.A. \& Jault, D., 2008. Quasi-geostrophic flows responsible for the secular variation of the Earth's magnetic field, Geophys. J. Int., 173, 421-443.

Palmer, T.N., Shutts, G.J. \& Swinbank, R., 1986. Alleviation of a systematic westerly bias in general circulation and numerical weather prediction models through an orographic gravity wave drag parametrization, $Q$. J.R. Meteorol. Soc., 112, 1001-1039.

Roberts, P.H. \& Aurnou, J.M., 2012. On the theory of core-mantle coupling, Geophys. astrophys. Fluid Dyn., 106, 157-230.

Schlaphorst, D., Thomas, C., Holme, R. \& Abreu, R., 2016. Investigation of core-mantle boundary topography and lowermost mantle with P4KP waves, Geophys. J. Int., 204, 1060-1071.

Taylor, J.B., 1963. The magnetohydrodynamics of a rotating fluid and the Earth's dynamo problem, Proc. R. Soc. A, 274, 274-283.

\section{APPENDIX A: MODES IN A HOMOGENEOUS FLUID PERMEATED BY A MAGNETIC FIELD PARALLEL TO THE BOUNDARY}

Expressions for the three vertical wavenumbers are derived in Section 3.1. This appendix presents the corresponding interior and boundarylayer modes. Examining first the interior solution $\left(\mathbf{u}_{1}, \mathbf{b}_{1}\right)$, Moffatt (1977) found

$u_{1, x}=A l b_{1, x}, \quad u_{1, y}=0, \quad b_{1, y}=-2 \mathrm{i} R o^{-1} A l^{2} b_{1, x}$, 
and $b_{1, y}=0$ in the asymptotic limit $R o^{-1} A l^{2} \rightarrow 0$ investigated here. There is no $y$-component for either $\mathbf{u}_{1}$ or $\mathbf{b}_{1}$ in the interior. We put together the matching conditions with the potential field in the solid region (10)

$\hat{b}_{1, z}+\hat{b}_{2, z}=\hat{\psi}$

$\hat{b}_{1, x}+\hat{b}_{2, x}=-\mathrm{i} \hat{\psi}$

the solenoidal condition $\left(\hat{b}_{x}+k_{z} \hat{b}_{z}=0\right)$ and the relation $\left(\left|k_{z, 2}\right| \gg\left|k_{z, 1}\right|\right)$. We deduce that $\hat{b}_{z, 2}$ is negligible $(O(\sqrt{Q}))$ relative to $\hat{b}_{z, 1}($ still at $z$ $=0)$. The solution in the fluid interior is

$\mathbf{b}_{1}=(\mathrm{i} \hat{\psi}, 0, \hat{\psi}) \exp (\mathrm{i} x+z)$

$\mathbf{u}_{1}=A l(\mathrm{i} \hat{\psi}, 0, \hat{\psi}) \exp (\mathrm{i} x+z)$.

Here, the choice for the sign of $\hat{\psi}$ differs from the choice made by Moffatt (1977) in his eq. (29) to ensure sign consistency from one section to the next in this article.

Turning to the boundary layer modes, they satisfy

$\hat{b}_{2 \pm, y}= \pm \mathrm{i} \hat{b}_{2 \pm, x}, \quad \hat{u}_{2 \pm, x}=\mathrm{i} \frac{R o}{2 A l} \hat{b}_{2 \pm, y}, \quad \hat{u}_{2 \pm, y}=-\mathrm{i} \frac{R o}{2 A l} \hat{b}_{2 \pm, x}$.

The modes labelled $(2+)$ and $(2-)$ multiply $\exp (z / 2 \sqrt{Q}) \exp (\mathrm{i} x)(\cos (z / 2 \sqrt{Q})+\mathrm{i} \sin (z / 2 \sqrt{Q})) \operatorname{and} \exp (z / 2 \sqrt{Q}) \exp (\mathrm{i} x)(\cos (z / 2 \sqrt{Q})-$ $\mathrm{i} \sin (z / 2 \sqrt{Q}))$, respectively. At $(z=0)$, the magnetic boundary condition $\hat{b}_{2, y}=0$ is satisfied if $\left(\hat{b}_{2+, y}+\hat{b}_{2-, y}=0\right)$. Then, from the first eq. (A4), we infer $\left(\hat{b}_{2+, x}=\hat{b}_{2-, x}\right)$ also at the fluid-solid interface. We combine this result with (A2) and (A3) to obtain

At $z=0, \quad \hat{b}_{2+, x}=\hat{b}_{2-, x}=-\mathrm{i} \hat{\psi}$,

$$
\begin{aligned}
\forall z \leq 0, \quad \mathbf{b}_{2}= & -2 \mathrm{i} \hat{\psi} \exp (z / 2 \sqrt{Q}) \exp (\mathrm{i} x) \\
& (\cos (z / 2 \sqrt{Q}),-\sin (z / 2 \sqrt{Q}),-\mathrm{i} \sqrt{Q}(\cos (z / 2 \sqrt{Q})+\sin (z / 2 \sqrt{Q}))), \\
\mathbf{u}_{2}= & \frac{R o}{A l} \hat{\psi} \exp (z / 2 \sqrt{Q}) \exp (\mathrm{i} x) \\
& (-\sin (z / 2 \sqrt{Q}),-\cos (z / 2 \sqrt{Q}),-\mathrm{i} \sqrt{Q}(\cos (z / 2 \sqrt{Q})-\sin (z / 2 \sqrt{Q}))) .
\end{aligned}
$$

The discrepancy between (A5) and the magnetic diffusion layer solution of Moffatt (1977) can be traced back to a sign error in his eq. (35).

\section{APPENDIX B: MODES IN A STRATIFIED FLUID PERMEATED BY A MAGNETIC FIELD TRANSVERSE TO THE BOUNDARY}

Expressions for the three vertical wavenumbers are derived in Section 4.1. This appendix discusses the three corresponding modes.

At leading order, the first mode $\left(\mathbf{u}_{1}, \mathbf{b}_{1}\right)$ is unaffected by diffusion in the $z$-direction. The mode wavenumber differs according to whether $\chi_{x} \equiv 0$ (f-plane) or $\chi_{x} \gg R o(\beta$-plane $)$. We have either

$\mathbf{u}_{1}=\gamma \mathbf{b}_{1}$,

when $\chi_{x} \equiv 0$ (with $\gamma$ defined by (49)) or

$\mathbf{u}_{1}=\mathrm{i} \gamma \sqrt{\frac{R o}{\chi_{x}}} \mathbf{b}_{1}$,

when $\chi_{x} \gg R o$. The two fields are almost aligned along $\mathbf{e}_{y}$, as there is geostrophic balance between the Coriolis force and the pressure force directed along $\mathbf{e}_{x}$ :

$\hat{u}_{1, x}=2 \mathrm{i} R_{m} \gamma^{2} F r^{2} Q\left(1-\frac{\chi_{x}}{R o}\right) \hat{u}_{1, y}$.

The second solution $\left(\mathbf{u}_{2}, \mathbf{b}_{2}\right)$ is very nearly a free magnetic mode inasmuch advection of the magnetic perturbation by $\mathbf{U}_{0}$ almost exactly balances diffusion. A very weak velocity perturbation suffices to force it. The induction equation gives

$\mathbf{u}_{2}=\frac{F r^{2} R_{m}}{\sqrt{Q R o}} \exp \left(\frac{\mathrm{i} \pi}{4}\right) \mathbf{b}_{2}$.

Then, from the axial vorticity equation, we find that the mode is mostly aligned along $\mathbf{e}_{x}$,

$\hat{b}_{2, y}=\frac{2 F r^{2}}{Q R o} \hat{b}_{2, x}$.

The character, kinetic or magnetic, of the third mode $\left(\mathbf{u}_{3}, \mathbf{b}_{3}\right)$ depends on the frequency ratio $F r / R o=\Omega / N$,

$\mathbf{b}_{3}=\mathrm{i} \frac{R_{m}}{k_{z, 3} A l} \mathbf{u}_{3}=-\exp \left(\frac{\mathrm{i} \pi}{4}\right) \frac{F r}{Q R o} \sqrt{\frac{R_{m}}{\gamma_{3}}} \mathbf{u}_{3}$. 
with

$k_{z, 3}=-\exp \left(\frac{\mathrm{i} \pi}{4}\right) \frac{\sqrt{R o Q}}{F r} \gamma_{3}, \quad \gamma_{3}^{2}=\frac{1+4 Q^{2}+Q^{2} \chi_{x}^{2}-4 \mathrm{i} Q^{3} \chi_{x}}{\left(1+4 Q^{2}\right)^{2}+Q^{2} \chi_{x}^{2}}$.

From the axial vorticity equation, we have also

$\hat{u}_{3, x}=\frac{1}{2 Q}\left(\mathrm{i} Q \chi_{x}-1\right) \hat{u}_{3, y}$.

The boundary conditions (11) for $b_{x}$ and $b_{z}$ involve the second and third modes. We have

$\hat{b}_{2, z}=\hat{\psi}, \quad \hat{b}_{3, z}=-\hat{\psi} \frac{F r}{\gamma_{3} \sqrt{Q R o}}\left(\mathrm{i} \gamma \sqrt{R_{m}}+\exp \left(\frac{\mathrm{i} \pi}{4}\right)\right)=-\mathrm{i} \hat{\psi} \frac{\gamma^{\prime} F r \sqrt{R_{m}}}{\gamma_{3} \sqrt{Q R o}}$

with

We obtain

$$
\gamma^{\prime}=\gamma\left(1+\exp \left(-\frac{\mathrm{i} \pi}{4}\right) \frac{1}{\sqrt{R_{m}}}\right)
$$

$\hat{\mathbf{b}}_{2}=\hat{\psi}\left(-\exp \left(-\frac{\mathrm{i} \pi}{4}\right) \gamma \sqrt{R_{m}},-2 \exp \left(-\frac{\mathrm{i} \pi}{4}\right) \frac{F r^{2} \gamma \sqrt{R_{m}}}{Q R o}, 1\right)$

$\hat{\mathbf{b}}_{3}=\gamma^{\prime} \sqrt{R_{m}} \hat{\psi}\left(\exp \left(-\frac{\mathrm{i} \pi}{4}\right),-2 \exp \left(-\frac{\mathrm{i} \pi}{4}\right) \frac{Q}{1-\mathrm{i} Q \chi_{x}},-\mathrm{i} \frac{F r}{\gamma_{3} \sqrt{Q R o}}\right)$.

The third magnetic boundary condition, on $\hat{b}_{y}$, determines $\hat{\mathbf{b}}_{1}$.

$\hat{\mathbf{b}}_{1}=2 \exp \left(-\frac{\mathrm{i} \pi}{4}\right) \frac{Q}{1-\mathrm{i} Q \chi_{x}} \gamma^{\prime} \sqrt{R_{m}} \hat{\psi} \mathbf{e}_{y}$.

Using the relationships (eqs B1 and B2) between the velocity and magnetic fields, we obtain

$\hat{\mathbf{u}}_{1}=2 \hat{\psi} \exp \left(-\frac{\mathrm{i} \pi}{4}\right) \gamma \gamma^{\prime} \sqrt{R_{m}} Q\left(2 \mathrm{i} R_{m} \gamma^{2} F r^{2} Q, 1,-2 \mathrm{i} \frac{\gamma Q F r^{2} \sqrt{R_{m}}}{\sqrt{Q R o}}\right)$,

when $\chi_{x} \equiv 0$ and

$\hat{\mathbf{u}}_{1}=2 \hat{\psi} \exp \left(\frac{\mathrm{i} \pi}{4}\right) \gamma \gamma^{\prime} \sqrt{\frac{R_{m} R o}{\chi_{x}}} \frac{Q}{1-\mathrm{i} Q \chi_{x}}\left(2 \mathrm{i} R_{m} \gamma^{2} F r^{2} Q, 1,-2 \mathrm{i} \frac{\gamma Q F r^{2} \sqrt{R_{m}}}{\sqrt{Q R o}}\right)$,

when $\chi_{x} \gg R o$. From eqs (B4) and (B6), we have also

$\hat{\mathbf{u}}_{2}=\hat{\psi} \frac{F r^{2} R_{m}}{\sqrt{Q R o}}\left(-\gamma R_{m}^{1 / 2}, 0, \exp \left(\frac{\mathrm{i} \pi}{4}\right)\right)$,

$\hat{\mathbf{u}}_{3}=\mathrm{i} \gamma^{\prime} \hat{\psi}\left(\frac{\gamma_{3} R o Q}{F r},-2 \frac{R o}{F r} \frac{\gamma_{3} Q^{2}}{1-\mathrm{i} Q \chi_{x}}, \exp \left(-\frac{\mathrm{i} \pi}{4}\right) \sqrt{Q R o}\right)$.

Thus, the no-penetration condition (9) involves only the third mode in the limit $F r^{2} / R o Q \ll 1$. In this limit, the main pressure term stems from the third mode

$\hat{p}=\frac{1}{F r^{2} k_{z, 3}} \hat{u}_{3, z}=\frac{\mathrm{i}}{F r^{2} k_{z, 3}} \hat{\zeta}$

[see expression (46) for $\hat{p}$ ] and the average pressure stress (13) along $x$ on the solid domain is

$\left\langle F_{p}\right\rangle=-\frac{1}{2} \frac{\Re\left(k_{z, 3}\right)}{F r^{2}\left|k_{z, 3}\right|^{2}}|\hat{\zeta}|^{2}$,

where $\mathfrak{R}(k)$ is the real part of $k$. We have:

$\frac{\Re\left(k_{z, 3}\right)}{\left|k_{z, 3}\right|}=-\frac{1}{\sqrt{2}}\left(1+\frac{4 Q^{2}\left(Q \chi_{x}\right)}{\left(1+\left(Q \chi_{x}\right)^{2}\right)^{1 / 2}\left(\left(1+4 Q^{2}\right)^{2}+\left(Q \chi_{x}\right)^{2}\right)^{1 / 2}}\right)^{1 / 2}$.

I write $k_{z, 3}^{2}$ as

$k_{z, 3}^{2}=\left(1+Q^{2} \chi_{x}^{2}\right) \frac{\operatorname{Ro} Q}{F r^{2}} \frac{g+\mathrm{i} f}{f^{2}+g^{2}}$

with

$g=4 Q^{2}\left(Q \chi_{x}\right), \quad f=1+4 Q^{2}+Q^{2} \chi_{x}^{2}$, 
$f^{2}+g^{2}=\left(1+Q^{2} \chi_{x}^{2}\right)\left(\left(1+4 Q^{2}\right)^{2}+\left(Q \chi_{x}\right)^{2}\right), \quad \frac{\Im\left(k_{z, 3}\right)}{\Re\left(k_{z, 3}\right)}=\frac{-g+\sqrt{f^{2}+g^{2}}}{f}$.

We have

$\left|k_{z, 3}\right|^{2}=\left(1+Q^{2} \chi_{x}^{2}\right) \frac{\operatorname{Ro} Q}{F r^{2}} \frac{1}{\sqrt{f^{2}+g^{2}}}$

and

$\left\langle F_{p}\right\rangle=-\frac{\sqrt{f^{2}+g^{2}}}{2 \operatorname{Ro} Q\left(1+Q^{2} \chi_{x}^{2}\right)} \Re\left(k_{z, 3}\right)|\hat{\zeta}|^{2}$.

After a few transformations, we can also write the pressure stress on the solid as

$\left\langle F_{p}\right\rangle=\frac{1}{2 \sqrt{2}} \frac{1}{F r \sqrt{R o Q} \sqrt{1+Q^{2} \chi_{x}^{2}}}\left(\sqrt{f^{2}+g^{2}}+g\right)^{1 / 2}|\hat{\zeta}|^{2}$.

The dominant term in the mean Coriolis force $\left\langle F_{C}\right\rangle$ acting on the fluid originates also from the third mode. Writing the $y$-component of the velocity perturbation as

$\hat{u}_{3, y}=2 \mathrm{i} \frac{Q}{1-\mathrm{i} Q \chi_{x}} k_{z, 3} \hat{\zeta}$

we have

$\left\langle F_{C}\right\rangle=-\frac{2 Q}{R o\left(1+Q^{2} \chi_{x}^{2}\right)}\left(\Im\left(k_{z, 3}\right)+Q \chi_{x} \Re\left(k_{z, 3}\right)\right)|\hat{\zeta}|^{2}$.

From (28), we calculate the mean magnetic force on the fluid. The boundary layer term (third mode) is dominant again. We find

$\left\langle F_{M, \mathcal{F}}\right\rangle=-\frac{\Im\left(k_{z, 3}\right)}{2 \operatorname{Ro} Q}|\hat{\zeta}|^{2}$.

We have

$$
\begin{aligned}
\left\langle F_{M, \mathcal{F}}\right\rangle+\left\langle F_{C}\right\rangle & =-\frac{\left(1+4 Q^{2}+Q^{2} \chi_{x}^{2}\right) \Im\left(k_{z, 3}\right)+4 Q^{3} \chi_{x} \Re\left(k_{z, 3}\right)}{2 \operatorname{Ro} Q\left(1+Q^{2} \chi_{x}^{2}\right)}|\hat{\zeta}|^{2}, \\
& =-\frac{\sqrt{f^{2}+g^{2}}}{2 \operatorname{Ro} Q\left(1+Q^{2} \chi_{x}^{2}\right)} \Re\left(k_{z, 3}\right)|\hat{\zeta}|^{2}
\end{aligned}
$$

Then, noting that (B22) is the expression for the pressure stress acting on the solid whereas (B27) is the expression for the total stresses acting on the fluid we verify that the total tangential stress on the fluid is zero, $\left\langle F_{M, \mathcal{F}}+F_{C}-F_{p}\right\rangle=0$.

Lastly, the dominant term (24) in the contribution of the Reynolds term to the mean stress is

$\frac{1}{2} \Re\left(k_{z, 3}\right)|\hat{\zeta}|^{2}$

It is much smaller than either $\left\langle F_{C}\right\rangle,\left\langle F_{M}\right\rangle$ or $\left\langle F_{p}\right\rangle$ (by a factor $R o Q$ ). The magnetic pressure stress is $O\left(R_{m}|\hat{\zeta}|^{2} /(R o Q)\right)$ and is thus negligible in comparison with $\left\langle F_{M}\right\rangle$ in the limit $F r^{2} / R o Q \rightarrow 0$.

\section{APPENDIX C: TRACTION ON A 2-D TOPOGRAPHY PERMEATED BY AN UNIFORM HORIZONTAL MAGNETIC FIELD}

This appendix gives the results necessary to the discussion in Section 5. First, the results of Section 3 and Appendix A are generalized to a single Fourier component of a 2-D topography:

$\zeta(x)=\Re\left(\hat{\zeta} \exp \left(i\left(x+k_{y} y\right)\right)\right)$,

with outward unit normal to the fluid region given by:

$\mathbf{n}=(0,0,1)-\Re\left(\mathrm{i} \hat{\zeta} \exp \left(\mathrm{i}\left(x+k_{y} y\right)\right)\right)\left(1, k_{y}, 0\right)$.

The solution in the fluid interior becomes

$\mathbf{b}_{1}=\left(\frac{\mathrm{i} \hat{\psi}}{k_{\|}}, \frac{\mathrm{i} k_{y} \hat{\psi}}{k_{\|}}, \hat{\psi}\right) \exp \left(\mathrm{i}\left(x+k_{y} y\right)+z\right)$,

$\mathbf{u}_{1}=\operatorname{Al}\left(\frac{\mathrm{i} \hat{\psi}}{k_{\|}}, \frac{\mathrm{i} k_{y} \hat{\psi}}{k_{\|}}, \hat{\psi}\right) \exp \left(\mathrm{i}\left(x+k_{y} y\right)+z\right)$,

$p=\frac{\mathrm{i}}{k_{\|} A l} \psi$, 
with $k_{\text {॥ }}$ given by (63). The boundary layer solution can be obtained as

$$
\begin{aligned}
\mathbf{b}_{2}= & \mathrm{i} \frac{\left(k_{\|}+1\right)}{k_{\|}} \hat{\psi} \exp \left(\frac{z}{2 \sqrt{Q}}\right) \exp \left(\mathrm{i}\left(x+k_{y} y\right)\right) \\
& \left(-\cos \left(\frac{z}{2 \sqrt{Q}}\right)-k_{y} \sin \left(\frac{z}{2 \sqrt{Q}}\right), \sin \left(\frac{z}{2 \sqrt{Q}}\right)-k_{y} \cos \left(\frac{z}{2 \sqrt{Q}}\right), \mathrm{i} k_{\|}^{2} \sqrt{Q}\left(\cos \left(\frac{z}{2 \sqrt{Q}}\right)+\sin \left(\frac{z}{2 \sqrt{Q}}\right)\right)\right), \\
\mathbf{u}_{2}= & -\frac{\left(k_{\|}+1\right)}{2 k_{\|}} \frac{R o}{A l} \hat{\psi} \exp \left(\frac{z}{2 \sqrt{Q}}\right) \exp \left(\mathrm{i}\left(x+k_{y} y\right)\right) \\
& \left(\sin \left(\frac{z}{2 \sqrt{Q}}\right)-k_{y} \cos \left(\frac{z}{2 \sqrt{Q}}\right), \cos \left(\frac{z}{2 \sqrt{Q}}\right)+k_{y} \sin \left(\frac{z}{2 \sqrt{Q}}\right), \mathrm{i} k_{\|}^{2} \sqrt{Q}\left(\cos \left(\frac{z}{2 \sqrt{Q}}\right)-\sin \left(\frac{z}{2 \sqrt{Q}}\right)\right)\right) .
\end{aligned}
$$

The horizontal velocity perturbation, at $z=0$, is parallel to the topography: the flow is directed along the valleys. Considering the $x$-averaged stress on the interface (C1) defined by a single Fourier component, we have

$$
\begin{aligned}
\left\langle F_{p}\right\rangle & =-\frac{1}{4 k_{\|} A l}\left(\psi \zeta^{*}+\psi^{*} \zeta\right) \\
\left\langle F_{p}-F_{p M}\right\rangle & =-\frac{\left(k_{\|}+1\right)}{4 k_{\|} A l}\left(\psi \zeta^{*}+\psi^{*} \zeta\right) .
\end{aligned}
$$

We can check again that the pressure stress on the fluid is exactly balanced by the Coriolis contribution $\left\langle F_{C}\right\rangle$. Since the flow perturbation next to the solid boundary is perpendicular to the wave vector $\left(i, k_{y}, 0\right)$, the tangential pressure stress is directed along the wave vector. Finally, the no-penetration condition (9) gives

$$
\hat{\psi}=\frac{i \hat{\zeta}}{A l\left(1-\frac{\mathrm{i} k_{\|}\left(k_{\|}+1\right)}{2 R_{m} \sqrt{Q}}\right)} .
$$

We note that $\hat{\psi}$ is independent of the sign of $k_{y}$.

We are now in the position to calculate the $x$-averaged traction exerted by the fluid pressure force on the topography defined by eq. (62). From the expression (C6) of the magnetic potential in the solid region, we derive :

$$
\begin{aligned}
\left\langle F_{p}-F_{p_{M}}\right\rangle & =-\frac{\left(k_{\|}+1\right)}{2 k_{\|}} \frac{\left(\hat{\psi} \hat{\zeta}^{*}+\hat{\psi}^{*} \hat{\zeta}\right)}{A l}\left(1+\cos \left(2 k_{y} y\right)\right) \\
& =\frac{2\left(k_{\|}+1\right)^{2} R_{m} \sqrt{Q}}{A l^{2}\left(\left(k_{\|}\left(k_{\|}+1\right)\right)^{2}+4 R_{m}^{2} Q\right)}\left(1+\cos \left(2 k_{y} y\right)\right)|\hat{\zeta}|^{2}
\end{aligned}
$$

